\title{
TM6SF2 rs58542926 Polymorphism is not Associated With Risk of Steatosis or Fibrosis in Chilean Patients With Chronic Hepatitis C
}

\author{
Alvaro Urzua,' Gabriel Mezzano, ${ }^{1}$ Javier Brahm, ${ }^{1}$ Jaime Poniachik, Julio Miranda, ${ }^{1}$ Dante D. Caceres, ${ }^{2}$ \\ Laura Carreno, ${ }^{3}$ and Mauricio Venegas ${ }^{1,}$, \\ ${ }^{1}$ Gastroenterology Section, Department of Medicine, University of Chile Clinical Hospital, Santiago, Chile \\ ${ }^{2}$ Environmental Health Program, School of Public Health, Faculty of Medicine, University of Chile \\ ${ }^{3}$ Department of Pathology, University of Chile Clinical Hospital, Santiago, Chile \\ "Corresponding author: Mauricio Venegas, 999 Santos Dumont Street, Independencia, Suite D3 359, Santiago, Chile. Tel: +56-229788348, Fax: +56-229788348, E-mail: \\ mvenegas@hcuch.cl
}

Received 2017 February 07; Revised 2017 March 09; Accepted 2017 March 25.

\begin{abstract}
Background: The polymorphism rs58542926 C > T (E167K) in the gene of transmembrane 6 superfamily member 2 (TM6SF2) has been identified as a determinant of hepatic steatosis and fibrosis in patients with non-alcoholic fatty liver disease. Only limited data have been published on this subject in chronic hepatitis $\mathrm{C}(\mathrm{CHC})$.

Objectives: This study aimed to evaluate the effect of TM6SF2 rs58542926 polymorphism on the risk of liver steatosis and fibrosis in Chilean patients with $\mathrm{CHC}$ infection.

Methods: A total of 153 biopsied CHC patients were genotyped for TM6SF2 rs58542926 using PCR-RFLP methodology. The risk of fatty liver was assessed by comparing absence $(<5 \%)$ with presence $(\geq 5 \%)$ of steatosis. The association with fibrosis was evaluated according to METAVIR score, by comparing patients in stage F0, F1, or F2 with patients in stage F3 or F4.

Results: TM6SF2 rs58542926 genotype CC was found in 138 (90.2\%) patients, whereas genotypes CT was found in 15 ( $9.8 \%$ ). No association was observed between rs58542926 genotype and risk of steatosis (OR 0.62, 95\% CI 0.17-2.22, $\mathrm{P}=0.459$ ) or fibrosis (OR 1.07, 95\% CI $0.29-3.87, \mathrm{P}=0.923$ ).

Conclusions: TM6SF2 rs58542926 polymorphism is not associated with the risk of liver steatosis or fibrosis in Chilean patients with CHC.
\end{abstract}

Keywords: HCV, Transmembrane 6 Superfamily Member 2, Non-Alcoholic Fatty Liver Disease

\section{Introduction}

Chronic infection with hepatitis $\mathrm{C}$ virus (HCV) is an important cause of morbidity and mortality (1). It is estimated that there are 185 million infected patients worldwide (2). Persistent HCV infection is associated with chronic hepatitis, liver cirrhosis, hepatocarcinoma development, liver failure, and death (3). The disease progression in chronic hepatitis $\mathrm{C}(\mathrm{CHC})$ infection is highly variable among individuals. Several factors have been recognized to be associated with the progression of HCVrelated liver fibrosis as well as clinical course, including age and time since initial HCV infection, alcoholism, obesity, co-infection with hepatitis B virus (HBV) and/or human immunodeficiency virus (HIV) and hepatic steatosis (4-6). Due to the fact that progression to fibrosis remains variable among patients with similar environmental or virological risks, host genetic predisposition has been suggested as another critical factor $(7,8)$.

A recent study has identified that non-synonymous polymorphism rs58542926 C > T (E167K) in the gene of transmembrane 6 superfamily member 2 (TM6SF2) is significantly associated with non-alcoholic fatty liver disease (NAFLD) (9). This TM6SF2 variant has subsequently been associated with severity of NAFLD-associated hepatic fibrosis/cirrhosis, independent of recognized confounding factors such as obesity, diabetes, age, or PNPLA3 (patatin-like phospholipase domain-containing 3) genotype (10).

To date, only three studies have examined the effects of the TMS6SF2 variant on CHC patients, bearing controversial results (11-13).

The aim of this investigation was to evaluate the association between TM6SF2 rs58542926 polymorphism and the risk of hepatic steatosis and fibrosis in Chilean patients with $\mathrm{CHC}$.

\section{Methods}

\subsection{Patient Samples}

We studied blood samples collected from 153 Chilean patients with CHC infection between March 2000 and Au- 
gust 2014 at University of Chile Clinical Hospital (Santiago, Chile). Inclusion criteria included: i) patients older than 18 years, ii) CHC diagnosis based on anti-HCV and HCV RNA positivity for at least 6 months, iii) availability of liver biopsy prior to antiviral treatment and, iv) availability of blood sample stored at $-80^{\circ} \mathrm{C}$ obtained concomitant with liver biopsy. Exclusion criteria included: i) alcohol abuse (> $30 \mathrm{~g} /$ day for males and $>20 \mathrm{~g} /$ day for females for at least 5 years), ii) autoimmune hepatitis, iii) co-infections with HIV or HBV and, iv) liver transplant recipients.

All patients planned to receive anti-viral therapy, and a liver biopsy was performed prior to treatment. Among these patients, no alcohol consumers and no co-infections with human immunodeficiency virus or hepatitis B virus were identified.

HCV genotyping was carried out by PCR-RFLP as previously described (14). The viral load was measured using the COBAS $₫$ TaqMan $®$ hepatitis $C$ virus test (Roche Molecular Systems, Branchburg, NJ, USA).

\subsection{Histological Classification}

The percentage of steatosis among total hepatocytes and fibrosis stages was assessed by a single pathologist who was blind to the study. The presence of steatosis was analyzed considering previous established criteria (15): Grade 0 (absence) < 5\%, Grade 15\% - 33\%, Grade 2 34\% - 66\%, and Grade $4>66 \%$. Due to the small number of patients in the grade 2 and 3 categories (only two patients in our study), we merely analyzed the steatosis as a qualitative variable $(<5 \%=$ absence, $\geq 5 \%=$ presence). Fibrosis was expressed according to the METAVIR scoring system (FO F4).

\subsection{TM6SF2 rs58542926 and PNPLA3 rs738409 Genotyping}

Genomic DNA was prepared from peripheral blood lymphocytes using high pure system nucleic acid kit (Roche Molecular Systems, Branchburg, NJ, USA). The TM6SF2 rs58542926 and PNPLA3 rs738409 polymorphisms genotyping was carried out by PCR-RFLP. For the PCR, oligonucleotide primers were designed with the aid of the NCBI Primer-Blast Tool. For TM6SF2, the primers were TM-F (5'- ACGGGGAAAGTTCAGGCACATTG -3') and TMR (5'- CCTGGGCAGCATGGTGAAACC -3'). For PNPLA3, the primers were PNPLA3-F (5'- AGTTCCCCGTTCTTTTGACCC -3') and PNPLA3-R (5'- TCAGCGCTAGCAGAGAAAGC -3'). The conditions for both PCR reactions ( $30 \mu \mathrm{L}$ ) were: initial denaturation at $94^{\circ} \mathrm{C}$ for 10 minutes, subsequently 40 cycles of: $94^{\circ} \mathrm{C}$ denaturation for 1 minute, $62^{\circ} \mathrm{C}$ annealing for $40 \mathrm{sec}$ onds, and $72^{\circ} \mathrm{C}$ extension for 1 minute, and a final extension for 10 minutes. The PCR product sizes were 429 and 247 base pairs for TM6SF2 and PNPLA3, respectively.
The RFLP assay for the TM6SF2 rs58542926 genotype was performed with $20 \mu \mathrm{L}$ of amplicons, which were digested with $5 \mathrm{U}$ of Hpy188I restriction endonuclease (New England Biolabs, MA, USA) at $37^{\circ} \mathrm{C}$ for 3 hours. Digestion of genotype CC with Hpy188I yields fragments of 178, 166, and 85 base pairs. Genotype TT digestion yields fragments of 251 and 178 base pairs, whereas genotype CT yields fragments of $251,178,166$, and 85 base pairs.

The RFLP assay for the PNPLA3 rs738409 genotype was also performed with $20 \mu \mathrm{L}$ of amplicons, which were digested with $5 \mathrm{U}$ of BtsCI restriction endonuclease (New England Biolabs) at $50^{\circ} \mathrm{C}$ for 3 hours. Digestion of genotype $\mathrm{CC}$ with BtsCI yielded the fragments of 173 and 74 base pairs, whereas genotype GG was not digested, keeping the original size. Both PCR and Restriction digestion products were analyzed on agarose gels stained with ethidium bromide and placed on a UV trans-illuminator for visualization and photographic record.

2.4. Association Between TM6SF2 rs58542926 C > T Polymorphism and Histological Damage

The association of TM6SF2 rs58542926 genotype (CC, CG and GG) with the risk of fibrosis was assessed by comparing patients with METAVIR score F0, F1, or F2 (no fibrosis, mild fibrosis, or intermediate fibrosis, respectively), with patients with METAVIR score F3 or F4 (severe fibrosis or cirrhosis). Steatosis was evaluated by comparing absence (< $5 \%$ ) versus presence ( $\geq 5 \%$ ).

The ethics committee of University of Chile Clinical Hospital approved this study with certificate of approval number 042/2015.

\subsection{Statistical Analysis}

Categorical variables were summarized as frequency and percentage, whereas continuous variables were shown as mean \pm standard deviation. Shapiro-Wilk test was used to evaluate normality. Categorical variables were analyzed using the two-sided chi-square test and quantitative variables were assayed by the nonparametric Kruskal-Wallis test or analysis of variance when appropriate. Multiple logistic regression models were performed to evaluate the association of TM6SF2 with hepatic steatosis and fibrosis through odd ratio (OR) risk effect measure, after adjustment for priori clinically relevant variables (age, gender, body mass index, diabetes, and PNPLA3 genotype). The software STATA version $\mathbf{1 2 . 0}$ was used for statistical analyses; $\mathrm{P}<0.05$ was considered statistically significant.

\section{Results}

153 Chilean patients with $\mathrm{CHC}$ were retrospectively analyzed in this cohort; 65 patients were male and the mean 
age was $51 \pm 11$ years. HCV genotype 1 was found in 127 patients (83\%), genotype 3 in 16 patients (10.5\%), genotype 2 in 4 patients (2.6\%), and genotypes 4 and 5 in 1 patient $(0.65 \%)$. The total number of patients with presence of steatosis was $80(52.3 \%)$ and with presence of severe fibrosis or cirrhosis was 55 (36\%).

The distribution of TM6SF2 rs58542926 genotypes was 138 patients with CC (90.2\%) and 15 with CT (9.8\%). No patient was found with homozygous genotype TT.

The 138 patients with TM6SF2 CC genotype were similar to the $15 \mathrm{CT}$ genotype patients with regard to the age, gender, body mass index (BMI), presence of diabetes, biochemical parameters, steatosis, fibrosis stage, and PNPLA3 genotype (Table 1).

In addition, we performed a univariable and multivariable logistic regression analysis for presence of steatosis and fibrosis that included the TM6SF2 rs58542926 genotype considering homozygote $\mathrm{CC}$ as a reference and involved reported clinical factors (Table 2). Age, BMI, and PNPLA3 GG genotype were significantly associated with the presence of steatosis using univariate analysis. After adjustment, only BMI and PNPLA3 GG genotype remained significantly associated with steatosis. On the other hand, age, presence of diabetes and steatosis were significantly associated with fibrosis on univariate analysis. After adjustment, only age and steatosis remained independently associated with histological liver damage(Table 2). TM6SF2 CT genotype was not associated with the risk of steatosis or fibrosis.

\section{Discussion}

This study demonstrated that TM6SF2 rs58542926 C > $\mathrm{T}$ polymorphism showed a low prevalence in Chilean patients with $\mathrm{CHC}$ and it was not associated with the risk of steatosis or fibrosis. Moreover, our current report represents the first analysis of this genetic variant in $\mathrm{CHC}$ from Latin-American region.

The role of TM6SF2 rs58542926 polymorphism as a determinant of steatosis and fibrosis has been clearly demonstrated in NAFLD $(9,10,16,17)$. Currently, only limited data have been published on the effect of TM6SF2 variants on $\mathrm{CHC}$, all of which are Italian studies that have obtained discordant results. Coppola et al. found an association between TM6SF2 and severe steatosis, but not with fibrosis in 148 patients with CHC having different HCV genotypes, predominantly of genotype 1 (11). Milano et al. described the association of TM6SF2 with steatosis and cirrhosis in a study of $815 \mathrm{CHC}$ patients, with different HCV genotypes although genotype 1 was the most prevalent (12). Conversely, Petta et al. concluded that TM6SF2 is not associated with steatosis and fibrosis in a cohort of 694 patients with $\mathrm{CHC}$,
Table 1. Characteristics of 153 Chilean Patients with Chronic Hepatitis C According to TM6SF2 rs58542926 Genotype ${ }^{\mathrm{a}}$

\begin{tabular}{|c|c|c|c|}
\hline Variable & $\begin{array}{c}\operatorname{TM6SF2} C C(n= \\
138)\end{array}$ & TM6SF2 CT $(n=15)$ & P Value \\
\hline Age, y & $51.4 \pm 10.9$ & $47.9 \pm 10.4$ & 0.2551 \\
\hline $\begin{array}{l}\text { Gender } \\
\text { (male/female) }\end{array}$ & $61 / 77$ & $4 / 11$ & 0.1920 \\
\hline Diabetes & $18(13)$ & $4(26.7)$ & 0.1530 \\
\hline $\begin{array}{l}\text { Body mass index, } \\
\mathrm{kg} / \mathrm{m}^{2}\end{array}$ & $26.46 \pm 4.22$ & $26.37 \pm 4.32$ & 0.9511 \\
\hline $\begin{array}{l}\text { Platelet counts } \times \\
10^{3} \mathrm{U} / \mathrm{L}\end{array}$ & $188 \pm 77$ & $196 \pm 98$ & 0.7660 \\
\hline ALT, IU/L & $120 \pm 100$ & $154 \pm 140$ & 0.5234 \\
\hline $\begin{array}{l}\text { Total cholesterol, } \\
\text { mg/dL }\end{array}$ & $171 \pm 29$ & $169 \pm 30$ & 0.8173 \\
\hline $\begin{array}{l}\text { HCV viral loads, } \\
\text { IU/mL }\end{array}$ & & & 0.9910 \\
\hline$>600.000$ & $83(60.1)$ & $9(60)$ & \\
\hline$<600.000$ & $55(39.9)$ & $6(40)$ & \\
\hline HCV genotype & & & 0.8760 \\
\hline 1 & $115(93.3)$ & $12(80)$ & \\
\hline 2 & $3(2.2)$ & $1(6.7)$ & \\
\hline 3 & $15(10.9)$ & $1(6.7)$ & \\
\hline 4 & $1(7.2)$ & & \\
\hline 5 & $1(7.2)$ & & \\
\hline $\begin{array}{l}\text { Not } \\
\text { determined }\end{array}$ & $3(2.2)$ & $1(6.7)$ & \\
\hline Steatosis, $\%$ & & & 0.3160 \\
\hline$<5$ & $64(46.4)$ & $9(60)$ & \\
\hline$\geq 5$ & $74(53.6)$ & $6(40)$ & \\
\hline \multicolumn{4}{|l|}{ Fibrosis } \\
\hline Fo - F2 & $88(63.8)$ & $10(66.7)$ & 0.8240 \\
\hline F3-F4 & $50(36.2)$ & $5(33.3)$ & \\
\hline PNPLA3 rs738409 & & & 1.000 \\
\hline $\mathrm{CC}$ & $32(23.2)$ & $3(20)$ & \\
\hline CG & $25(18.1)$ & $3(20)$ & \\
\hline GG & $72(52.2)$ & $7(46.7)$ & \\
\hline $\begin{array}{l}\text { Not } \\
\text { determined }\end{array}$ & $9(6.5)$ & $2(13.3)$ & \\
\hline
\end{tabular}

Abbreviation: ALT, alanine aminotransferase.

${ }^{\mathrm{a}}$ Values are expressed as mean \pm SD or No. (\%).

all with HCV genotype 1 (13). Our results in CHC (containing mainly HCV genotype 1) are consistent with the latter study. Interestingly, the three mentioned studies reported the prevalence of TM6SF2 rs58542926 C > T polymorphism as low as we found in the current study, indicating that the 
Table 2. Univariable and Multivariable Logistic Regression Analysis of TM6SF2 rs58542926 Genotype Influence on Histological Liver Damage Characteristics ${ }^{\mathrm{a}}$

\begin{tabular}{|c|c|c|c|c|c|c|}
\hline \multirow[b]{2}{*}{ Variables } & \multicolumn{3}{|c|}{ Univariate } & \multicolumn{3}{|c|}{ Multivariable } \\
\hline & OR & 95\% CI & P Value & OR & 95\% CI & P Value \\
\hline \multicolumn{7}{|l|}{ Steatosis $(<5 \%$ versus $\geq \% 5)$} \\
\hline TM6SF2 rs58542926 CC & 1.00 & & & & & \\
\hline TM6SF2 rs58542926 CT & 0.58 & $0.19-1.71$ & 0.320 & 0.62 & $0.17-2.22$ & 0.459 \\
\hline Age, $y$ & 1.04 & $1.01-1.07$ & 0.019 & 1.02 & $0.98-1.05$ & 0.346 \\
\hline Sex (Female) & 0.81 & $0.43-1.54$ & 0.516 & 0.81 & $0.38-1.74$ & 0.598 \\
\hline BMI, $\mathrm{Kg} / \mathrm{m}^{2}$ & 1.15 & $1.06-1.26$ & $0.001^{b}$ & 1.16 & $1.05-1.27$ & $0.003^{\mathrm{b}}$ \\
\hline Diabetes & 2.18 & $0.83-5.68$ & 0.113 & 1.88 & $0.59-5.94$ & 0.283 \\
\hline PNPLA3 rs738409 GG & 3.13 & $1.36-7.20$ & $0.007^{\mathrm{b}}$ & 3.57 & $1.43-8.89$ & $0.006^{\mathrm{b}}$ \\
\hline \multicolumn{7}{|l|}{ Fibrosis (Fo-F2 versus F3-F4) } \\
\hline TM6SF2 rs58542926 CC & 1.00 & & & & & \\
\hline TM6SF2 rs58542926 CT & 0.88 & $0.28-2.72$ & 0.824 & 1.07 & $0.29-3.87$ & 0.923 \\
\hline Age, y & 1.06 & $1.02-1.09$ & $0.002^{\mathrm{b}}$ & 1.05 & $1.01-1.09$ & $0.011^{\mathrm{b}}$ \\
\hline Sex (Female) & 1.21 & $0.62-2.35$ & 0.578 & 1.59 & $0.74-3.44$ & 0.235 \\
\hline $\mathrm{BMI}, \mathrm{Kg} / \mathrm{m}^{2}$ & 1.08 & $0.99-1.17$ & 0.051 & 1.03 & $0.94-1.13$ & 0.486 \\
\hline Diabetes & 3.84 & $1.49-9.87$ & $0.005^{\mathrm{b}}$ & 2.43 & $0.88-6.69$ & 0.085 \\
\hline Steatosis ( $\geq \% 5)$ & 2.63 & $1.32-4.24$ & $0.006^{\mathrm{b}}$ & 2.17 & $1.02-4.69$ & $0.043^{\mathrm{b}}$ \\
\hline
\end{tabular}

Abbreviations: BMI, body mass index; $\mathrm{CI}$, confidence interval; OR, odds ratio.

${ }^{\mathrm{a}}$ Values are expressed as mean $\pm \mathrm{SD}$ or No. (\%).

${ }^{\mathrm{b}} \mathrm{P}$ value $<0.05$ is considered statistically significant.

majority of the patients are heterozygous which makes it difficult to determine the possible effect among homozygous TT patients. Similarly, studies on patients with NAFLD and without HCV infection also report very low prevalence of homozygous patients with unfavorable T allele $(9,10)$.

On the other hand, in our cohort, only $10.5 \%$ were infected by HCV genotype 3 strains and no chronic alcohol consumption was reported; thus, these factors are known to be associated with steatosis and do not represent a significant risk for fatty liver. Therefore, PNPLA3 GG genotype and BMI were the only factors associated with the presence of steatosis, which is consistent with previous studies (18, 19). Regarding the PNPLA3 polymorphism, it is necessary to note that more than $50 \%$ of our patients were homozygotes GG, which could explain in part the observed steatosis. This percentage is much higher than that described in the Italian studies mentioned above $(<10 \%)$.

It is known that fat accumulation is associated with oxidative hepatocellular damage, inflammation, and activation of fibrogenesis, which can progress to cirrhosis and hepatocellular carcinoma (20). In our analysis, we actually found a clear association between the presence of steatosis and fibrosis stage.

In conclusion, in this study the TM6SF2 rs58542926
C > T polymorphism had low prevalence in Chilean patients with $\mathrm{CHC}$ and was not associated with risk of hepatic steatosis or liver fibrosis. Further Latin-American and worldwide studies are needed to confirm these findings in different populations.

\section{Footnotes}

Financial Disclosure: None to declare.

Funding/Support: This study was funded by grant OAIC 747/15 from University of Chile Clinical Hospital (Prof. Venegas).

\section{References}

1. Cooke GS, Lemoine M, Thursz M, Gore C, Swan T, Kamarulzaman A, et al. Viral hepatitis and the Global Burden of Disease: a need to regroup. J Viral Hepat. 2013;20(9):600-1. doi: 10.1111/jvh.12123. [PubMed: 23910643].

2. Mohd Hanafiah K, Groeger J, Flaxman AD, Wiersma ST. Global epidemiology of hepatitis $C$ virus infection: new estimates of agespecific antibody to HCV seroprevalence. Hepatology. 2013;57(4):133342. doi: 10.1002/hep.26141. [PubMed: 23172780].

3. Lauer GM, Walker BD. Hepatitis C virus infection. $N$ Engl J Med. 2001;345(1):41-52. doi: 10.1056/NEJM200107053450107. [PubMed: 11439948]. 
4. Huang CF, Chuang WL, Yu ML. Chronic hepatitis C infection in the elderly. Kaohsiung J Med Sci. 2011;27(12):533-7. doi: 10.1016/j.kjms.2011.10.020. [PubMed: 22208535].

5. Poynard T, Bedossa P, Opolon P. Natural history of liver fibrosis progression in patients with chronic hepatitis C. The OBSVIRC METAVIR, CLINIVIR, and DOSVIRC groups. Lancet. 1997;349(9055):82532. [PubMed: 9121257].

6. Wright M, Goldin R, Fabre A, Lloyd J, Thomas H, Trepo C, et al. Measurement and determinants of the natural history of liver fibrosis in hepatitis $C$ virus infection: a cross sectional and longitudinal study. Gut. 2003;52(4):574-9. [PubMed: 12631672].

7. Missiha SB, Ostrowski M, Heathcote EJ. Disease progression in chronic hepatitis C: modifiable and nonmodifiable factors. Gastroenterology. 2008;134(6):1699-714. doi: 10.1053/j.gastro.2008.02.069. [PubMed: 18471548].

8. Patin E, Kutalik Z, Guergnon J, Bibert S, Nalpas B, Jouanguy E, et al. Genome-wide association study identifies variants associated with progression of liver fibrosis from HCV infection. Gastroenterology 2012;143(5):1244-52 e1-12. doi: 10.1053/j.gastro.2012.07.097. [PubMed: 22841784].

9. Kozlitina J, Smagris E, Stender S, Nordestgaard BG, Zhou HH, TybjaergHansen A, et al. Exome-wide association study identifies a TM6SF2 variant that confers susceptibility to nonalcoholic fatty liver disease. Nat Genet. 2014;46(4):352-6. doi: 10.1038/ng.2901. [PubMed: 24531328]

10. Liu YL, Reeves HL, Burt AD, Tiniakos D, McPherson S, Leathart JB, et al. TM6SF2 rs58542926 influences hepatic fibrosis progression in patients with non-alcoholic fatty liver disease. Nat Commun. 2014;5:4309. doi: 10.1038/ncomms5309. [PubMed: 24978903].

11. Coppola N, Rosa Z, Cirillo G, Stanzione M, Macera M, Boemio A, et al. TM6SF2 E167K variant is associated with severe steatosis in chronic hepatitis C, regardless of PNPLA3 polymorphism. Liver Int 2015;35(8):1959-63. doi: 10.1111/liv.12781. [PubMed: 25581573].

12. Milano M, Aghemo A, Mancina RM, Fischer J, Dongiovanni P, De Nicola $S$, et al. Transmembrane 6 superfamily member 2 gene E167K variant impacts on steatosis and liver damage in chronic hepatitis $\mathrm{C}$ patients. Hepatology. 2015;62(1):111-7. doi: 10.1002/hep.27811. [PubMed
25820484].

13. Petta S, Maida M, Grimaudo S, Pipitone RM, Macaluso FS, Cabibi D, et al. TM6SF2 rs58542926 is not associated with steatosis and fibrosis in large cohort of patients with genotype 1 chronic hepatitis C. Liver Int. 2016;36(2):198-204. doi: 10.1111/liv.12918. [PubMed: 26259026].

14. Thiers V, Jaffredo F, Tuveri R, Chodan N, Brechot C. Development of a simple restriction fragment length polymorphism(RFLP) based assay for HCV genotyping and comparative analysis with genotyping and serotyping tests.J Virol Methods. 1997;65(1):9-17. [PubMed: 9128857].

15. Kleiner DE, Brunt EM, Van Natta M, Behling C, Contos MJ, Cummings OW, et al. Design and validation of a histological scoring system for nonalcoholic fatty liver disease. Hepatology. 2005;41(6):1313-21. doi: 10.1002/hep.20701. [PubMed: 15915461].

16. Dongiovanni P, Petta S, Maglio C, Fracanzani AL, Pipitone R, Mozzi E, et al. Transmembrane 6 superfamily member 2 gene variant disentangles nonalcoholic steatohepatitis from cardiovascular disease. Hepatology. 2015;61(2):506-14. doi: 10.1002/hep.27490. [PubMed: 25251399].

17. Sookoian S, Castano GO, Scian R, Mallardi P, Fernandez Gianotti T, Burgueno AL, et al. Genetic variation in transmembrane 6 superfamily member 2 and the risk of nonalcoholic fatty liver disease and histological disease severity. Hepatology. 2015;61(2):515-25. doi: 10.1002/hep.27556. [PubMed: 25302781].

18. Trepo E, Pradat P, Potthoff A, Momozawa Y, Quertinmont E, Gustot $\mathrm{T}$, et al. Impact of patatin-like phospholipase-3 (rs738409 C>G) polymorphism on fibrosis progression and steatosis in chronic hepatitis C. Hepatology. 2011;54(1):60-9. doi: 10.1002/hep.24350. [PubMed: 21488075].

19. Petta S, Vanni E, Bugianesi E, Rosso C, Cabibi D, Camma C, et al. PNPLA3 rs738409 I748M is associated with steatohepatitis in 434 non-obese subjects with hepatitis C. Aliment Pharmacol Ther. 2015;41(10):939-48. doi: 10.1111/apt.13169. [PubMed: 25801076].

20. Vernon G, Baranova A, Younossi ZM. Systematic review: the epidemiology and natural history of non-alcoholic fatty liver disease and non-alcoholic steatohepatitis in adults. Aliment Pharmacol Ther. 2011;34(3):274-85. doi: 10.1111/j.1365-2036.2011.04724.x. [PubMed: 21623852]. 